\title{
Distributed MIMO in Multi-Cell Wireless Systems via Finite-Capacity Links
}

\author{
Osvaldo Simeone \\ CWCSPR, ECE Department \\ New Jersey Institute of Technology \\ Newark, NJ 07102 \\ osvaldo.simeone@njit.edu
}

\author{
Oren Somekh and H. Vincent Poor \\ Department of Electrical Engineering \\ Princeton University \\ Princeton, NJ 08544 \\ \{orens, poor\}@princeton.edu
}

\author{
Shlomo Shamai (Shitz) \\ Department of Electrical Engineering \\ Technion \\ Haifa, 32000, Israel \\ sshlomo@ee.technion.ac.il
}

\begin{abstract}
Infrastructure networks, such as cellular systems, are expected to benefit from both multicell processing (cooperation at the base station level) and relaying (cooperation at the user level). This paper provides a brief review of recent results concerning the analysis of such technologies, under the practical assumption that both multicell processing and user cooperation are enabled by error-free but limited-capacity inter-base station and inter-user links, respectively. The focus is on non-fading uplink and downlink channels based on Wyner-type cellular models. The analytical treatment enhances the insight into the potential and the limitations imposed by capacity constraints on the performance gains provided by cooperative (distributed MIMO) techniques.
\end{abstract}

\section{INTRODUCTION}

A key technology that has been identified to potentially overcome the limitations of conventional cellular wireless networks, in terms of throughput and coverage is cooperation, to be deployed at either the base station (BS) or mobile station (MS) levels. As far as the BS level is concerned, multi-cell processing (MCP), sometimes referred to also as "distributed antenna system", prescribes joint encoding/ decoding of the signals transmitted/ received at the BSs via the high-capacity backhaul connecting the BSs (see [1] [2] for recent surveys on MCP). Cooperation at the MS level in the context of cellular networks has been studied under different names, such as mesh, hybrid or multi-hop cellular networks, and is based on specific forms of relaying by the MSs (see, e.g., [3]).

BS and MS cooperative technologies are enabled by the presence, respectively, of inter-BS (backhaul) and inter-MS links that are not exploited by conventional cellular systems for the purpose of encoding or decoding. These links can be either wireless, orthogonal or not, thus possibly affecting the interference or bandwidth budget of the network, or wired, thus possibly requiring additional deployment efforts.

Analysis of MCP (i.e., BS cooperation) has been so far mostly based on the assumption that all the BSs in the network are connected to a central processor via links of unlimited capacity. In this case, the set of BSs effectively acts as a multiantenna transmitter (downlink) or receiver (uplink) with the caveat that the antennas are geographically distributed over a large area. Since the assumption of unlimited-capacity links to a central processor is quite unrealistic for large networks, more recently, there have been attempts to alleviate this condition by considering alternative models. In [4] a model is studied where only a subset of neighboring cells is connected to the same central unit for joint processing. In [8] [9] a topological constraint is imposed in that there exist links only between adjacent cells, and message passing techniques are implemented in order to perform joint decoding in uplink. Finally, reference [10] focuses on the uplink and assumes that the links between all the BSs and a central processor have finite capacity (finite-capacity backhaul). The reader is referred to [5][6][7] for a different framework which deals with practical aspects of finite-capacity backhaul cellular systems incorporating MCP.

Information-theoretic analysis of MS cooperation in cellular networks is a more recent development. References include [11] [12] where the uplink of a two-hop mesh network is studied with amplify-and-forward (AF) cooperation (half-duplex and full-duplex, respectively) and [13] (half-duplex) [14] (fullduplex) where decode-and-forward (DF) cooperation is investigated (a thorough tutorial on cooperation techniques can be found in [15]).

Most of the analysis on MCP is based on different variants of a simple and analytically tractable model for cellular system proposed by Wyner [16] (henceforth, the Wyner model, see also [17]). Accordingly, the cells are arranged in either an infinite linear array or in the more familiar twodimensional hexagonal pattern, and only adjacent-cell interference is present and characterized by a single gain parameter $\alpha \in(0,1]$. In some cases, we will also refer to a variation of the regular Wyner model, called soft-handoff model, where, assuming a linear geometry, MSs are located at the border between two successive cells and thus communicate only with the two corresponding BSs (see Fig. 1). This model has been proposed in [20] and later adopted in a number of works [18]-[22]. With simplicity and analytical tractability in mind, the Wyner model provides perhaps the simplest framework for a cellular system that still captures the essence real-life phenomena such as intercell interference and fading.

In this presentation, we focus on cellular systems abstracted according to Wyner-type models and study the impact of finitecapacity links on both MCP (finite-capacity backhaul) and MS cooperation (conferencing) for the uplink and the downlink. 


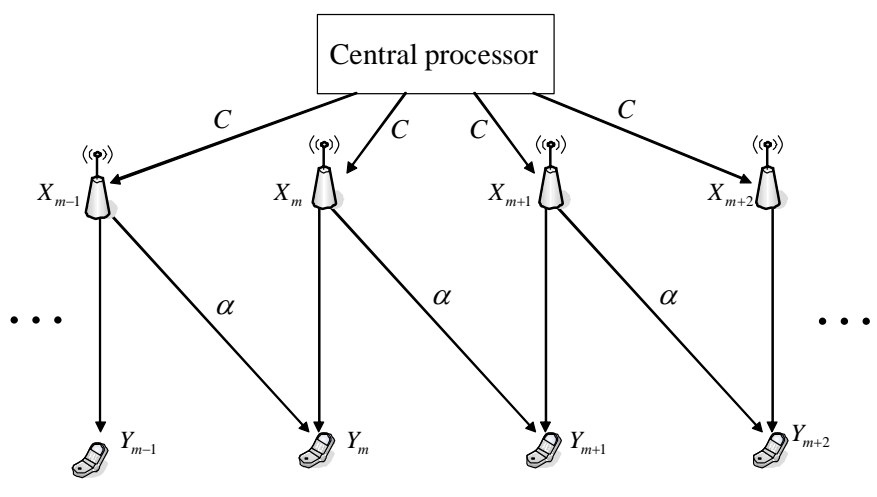

Fig. 1. Downlink of a soft-handoff model with finite-capacity backhaul.

\section{MCP WITH LIMITED-CAPACITY BACKHAUL}

Analysis of MCP with limited-capacity backhaul can be carried out according to different assumptions regarding the knowledge of codebooks (or, more generally, encoding functions) at the BSs (codebook information, CI). In [10] [23], the uplink of a Wyner model with MCP and limited-capacity backhaul was studied in two scenarios: (i) the BSs are oblivious to the codebooks used by the MSs (i.e., no CI) so that decoding is exclusively performed at the central processor; (ii) the BSs are aware of the codebooks used by the local and the nearby MSs (cluster CI).

Here we briefly discuss the downlink scenario with finitecapacity backhaul, which is more thoroughly investigated in [27]. Similarly to [10][23], three scenarios are considered that present different trade-offs between global processing at the central unit and local processing at the BSs, and different requirements in terms of $\mathrm{CI}$ at the BSs: (a) local encoding with CI limited to a subset of adjacent BSs (cluster CI); (b) mixed local and central encoding with only local CI; (c) central encoding with oblivious cells (no CI). Three transmission strategies are proposed that provide achievable rates for the considered scenarios.

Let us start with the case of cluster CI (case (a)). Exploiting the local interference structure of the soft-handoff setup, shutting off one every $(J+2)$ th BSs forms isolated clusters of $J$ cells (see also [36]). Each BS is aware of the codebooks of its cluster's users, while the central unit sends each cluster's messages to all its BSs via the limited capacity links. Having the cluster's CI and messages, each BS performs a form of DPC locally (under individual equal per BS power constraint [29]) and transmits its signal accordingly. In [27] two cluster DPC schemes are considered: 1) sequential encoding in which each BS invokes DPC to cancel the interfering signal coming from its left neighboring BS; and 2) joint encoding in which each BS performs optimal joint DPC within the cluster. It is shown in [27] that the per-cell rate of the joint encoding scheme, although it approaches the cut-set bound when both the limited capacity $C$ and the cluster size $J$ go to infinity while their ratio converges to some finite constant, is in general smaller than the rate of sequential encoding for relatively small

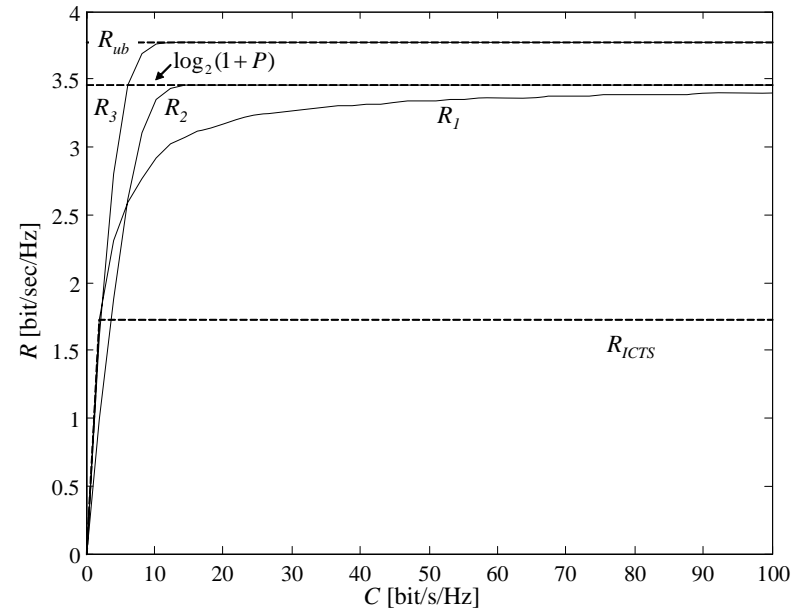

Fig. 2. Downlink with finite-capacity backbone: Rates achievable with ICTS ( $\left.R_{I C T S}\right)$, with local processing and cluster CI $\left(R_{1}\right)$, with mixed processing and local CI $\left(R_{2}\right)$ and with central processing and no CI $\left(R_{3}\right)$ versus $C$ for $P=10 d B$ and $\alpha=1$.

values of $C$.

In case (b) of local CI, a scheme is proposed in [27] whereby each BS receives from the central unit through the limited capacity link its local user's message and a quantized version of the signal to be transmitted by its left neighboring BS. By performing local DPC, each BS is then able to cancel the interfering signal coming from its left neighboring BS. The per-cell rate of this scheme is given in [27] as the unique solution of a fixed-point equation. The closed-form expression derived in [27] reveals that the rate approaches the cut-set bound only when the SNR $P$ is high and the interference level is low.

With oblivious BSs (case (c)), joint DPC under individual power constraint is performed by the central unit, which sends quantized versions of the transmitted signals to the BSs via the limited-capacity links. Since the transmitted quantization noise decreases the overall SNR seen by the MSs, joint DPC is designed to meet lower SNR values and tighter power constraints than those of the unlimited setup [20]. As expected, the resulting per-cell rate is shown in [27] to approach the cutset bound with increasing $C$. Moreover, also in the high-SNR regime the scheme performs well achieving rates which are less than 1 [bit/ channel use] below the cut-set bound.

Fig. 2 shows the rates achievable by local processing and cluster CI $R_{1}$ with optimized cluster-size $J$ and sequential DPC, by mixed processing and local CI $R_{2}$, and by central processing and no CI $R_{3}$, versus the backhaul capacity $C$ for $P=10[\mathrm{~dB}]$ and $\alpha=1$. Also shown is the rate achievable with Inter Cell Time Sharing (ICTS), which prescribes simple interference-free transmission via frequency reuse (i.e., even and odd- numbered cells are operated alternatively at different times) [2]. It is noted that the optimal cluster-size $J$ is increasing with the capacity $C$ (not shown). It is seen that if $C$ is large enough, and for relatively small to moderate values of 
$P$, scheme 3 , which performs central processing with oblivious $\mathrm{BSs}$, is to be preferred.

The main conclusions of [27] is that central processing, even with oblivious BSs, is the preferred choice for small-tomoderate SNRs or when the backhaul capacity $C$ is allowed to increase with the SNR $P$. On the other hand, for high SNR values and fixed capacity $C$, a system with oblivious BSs is limited by the quantization noise, and knowledge of the codebooks at the BSs becomes the factor dominating the performance. Therefore, in this regime, transmission schemes characterized by local CI or cluster CI coupled with local processing allow to achieve better performance than central processing with oblivious cells.

\section{CONFERENCING WITH LIMITED-CAPACITY INTER-MS LINKS}

We now direct attention to a scenario where BSs perform multi-cell processing (here, with infinite-capacity backhaul), while the MSs are allowed to cooperate over finite-capacity links (see Fig. 3). These links should be considered as additional spectral resources (orthogonal to the main uplink or downlink channel) that are available to enable cooperation. In modeling the interaction among MSs, the framework of conferencing encoders for the uplink [33] (see also [34] [35] for related scenarios) and decoders for the downlink [30]-[32] is followed. Moreover, we focus on a scenarios with intra-cell TDMA so that conferencing channels exist only between MSs belonging to adjacent cells (inter-cell conferencing) (reference [28] also considers intra-cell conferencing for the uplink).

Starting with the uplink and multicell decoding, an upper bound on the per-cell rate is obtained by considering a system with perfect inter-MS cooperation whereby all the MSs are able to exchange the local messages with all the other active MSs in the network. The system at hand is thus equivalent to an ISI channel with CSI at the transmitter (or equivalently an infinite MIMO system with a Toeplitz channel matrix), for which a stationary input with power spectral density obtained via standard waterfilling is known to be optimal (in terms of sum-rate and thus, by symmetry of the system at hand, in terms of per-cell rate). An achievable rate can be derived by considering an extension of the approach in [33] to multiple sources (in the spirit of [37], Sec. VII) [28]. Specifically, rate splitting is performed at each MS so that one message (the common message) is communicated to the $2 K$ nearby MSs ( $K$ on either side) in $K$ rounds of conferencing on the limitedcapacity links, so that in the transmission phase cooperative transmission by $2 K+1$ MSs can take place for every common message. Based on the observation that a stationary input is asymptotically optimal, cooperative transmission can be designed so as to implement an equivalent linear pre-filtering of the transmitted signal, which in the limit of $C \rightarrow \infty$ (and $K \rightarrow \infty$ ) allows the upper bound discussed above to be attained via appropriate design of the filter at hand [28].

In Fig. 4 the achievable rate $R$ is plotted versus the inter-cell gain $\alpha$ along with the lower bound $R_{\text {lower }}$ (no cooperation) and upper bound $R_{\text {upper }}$ for $C=10$ and $P=3$ [dB]. It can

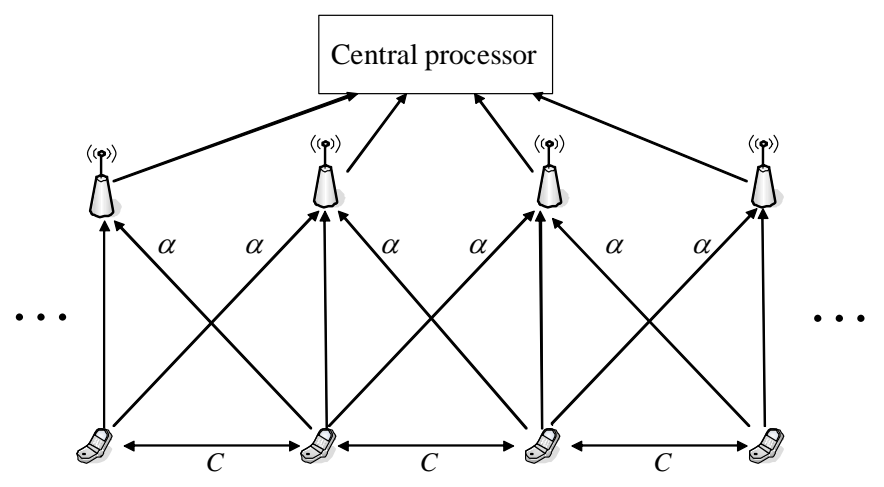

Fig. 3. Uplink with finite-capacity inter-MS (conferencing) links.

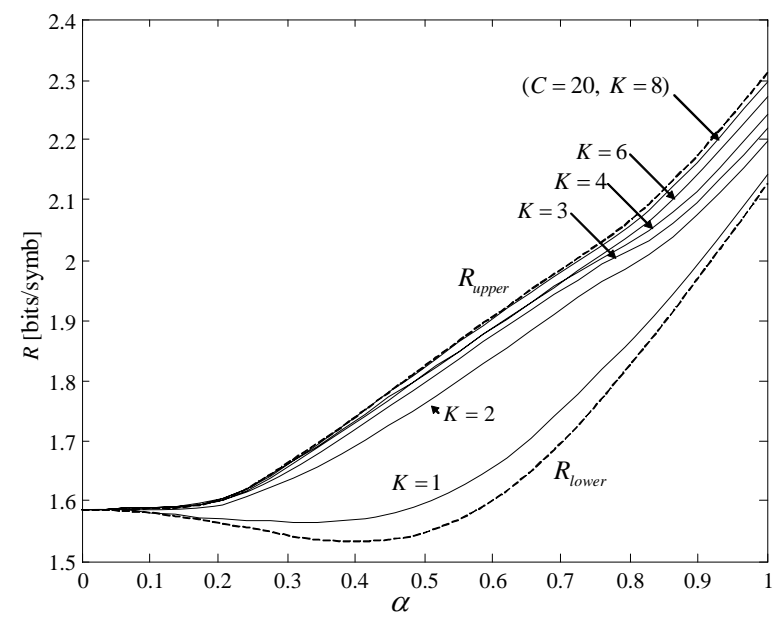

Fig. 4. Uplink with finite-capacity inter-MS links: Achievable rate with intercell conferencing and intra-cell TDMA versus the inter-cell gain $\alpha$. Lower and upper bounds are also shown for reference $(C=10$ and $P=3[\mathrm{~dB}])$.

be seen that very relevant performance gains can be harnessed by increasing the number of conference rounds, especially from $K=1$ to $K=2$. Moreover, having sufficient large conferencing capacity $C$ and number of conference rounds $K$ (with $C / K \geq R_{\text {upper }}$ ) enables for the upper bound to be approached. It is worth mentioning that increasing $K$ is always beneficial to obtain a better approximation of the waterfilling strategy. However, due to the finite conferencing capacity $C$, it is not necessarily advantageous in terms of the achievable rate (not shown).

\section{CONCLUding Remarks}

The idea of creating multiple antenna (MIMO) transceivers by exploiting finite capacity links between distributed antennas has been considered in this paper in the context of multicell cellular systems. Information-theoretic results concerning both cooperation among BSs and MSs have been reviewed, providing insight into performance and design choices. Open problems include the analysis of fading channels with different degrees of CSI at the MSs and BSs. 


\section{ACKNOWLEDGMENT}

The research was supported in part by a Marie Curie Outgoing International Fellowship and the NEWCOM++ network of excellence both within the 6th European Community Framework Programme, by the REMON Consortium and by the U.S. National Science Foundation under Grants CNS-0625637, CNS-06-26611, and ANI-03-38807.

\section{REFERENCES}

[1] S. Shamai (Shitz), O. Somekh, and B. M. Zaidel, "Multi-cell communications: An information theoretic perspective," in Proc. of the Joint Workshop on Commun. and Coding (JWCC'04), Donnini, Florence, Italy, Oct.14-17, 2004.

[2] O. Somekh, O. Simeone, Y. Bar-Ness, A. M. Haimovich, U. Spagnolini, and S. Shamai (Shitz), Distributed Antenna Systems: Open Architecture for Future Wireless Communications, ch. An Information Theoretic View of Distributed Antenna Processing in Cellular Systems, Auerbach Publications, CRC Press, May 2007.

[3] R. Pabst, B. H. Walke, D. C. Schultz, P. Herhold, H. Yanikomeroglu, S. Mukherjee, H. Viswanathan, M. Lott, W. Z. Sirwas, D. D. Falconer and G. P. Fettweis, "Relay-based deployment concepts for wireless and mobile broadband radio,” IEEE Commun. Magazine, pp. 80-89, Sept. 2004.

[4] O. Somekh, B. M. Zaidel and S. Shamai (Shitz), "Spectral efficiency of joint multiple cell-site processors for randomly spread DS-CDMA systems,” IEEE Trans. Inform. Theory, vol. 52, no. 7, pp. 2625-2637, Jul. 2007.

[5] P. Marsch, S. Khattak, and G. Fettweis, "A framework for determining realistic capacity bounds for distributed antenna systems," in Proc. IEEE Inform. Theory Workshop (ITW'06), China, Oct. 2006.

[6] P. Marsch and G. Fettweis, "A framework for optimizing the downlink performance of distributed antenna systems under a constrained backhaul," in Proc. European Wireless Conf. (EW 2007), April 1-4, Paris, France, 2007.

[7] P. Marsch and G. Fettweis, "A framework for optimizing the uplink performance of distributed antenna systems under a constrained backhaul," in Proc. IEEE International Conference on Communications (ICC 2007), Jun. 24-28, Glasgow, Scotland, 2007.

[8] E. Atkas, J. Evans and S. Hanly, "Distributed decoding in a cellular multiple access channel," in Proc. IEEE International Symposium on Inform. Theory (ISIT 2004), p. 484, June. 27-Jul. 2, Chicago, Illinois, 2004.

[9] O. Shental, A. J. Weiss, N. Shental and Y. Weiss, "Generalized belief propagation receiver for near optimal detection of two-dimensional channels with memory," in Proc. Inform. Theory Workshop (ITW 2004), San Antonio, Texas, Oct. 24-29, 2004.

[10] A. Sanderovich, O. Somekh and S. Shamai (Shitz), "Uplink macro diversity with limited backhaul capacity,” in Proc. IEEE International Symposium on Inform. Theory (ISIT 2007), Nice, France, June 24-29, 2007.

[11] O. Simeone, O. Somekh, Y. Bar-Ness and U. Spagnolini, "Uplink throughput of TDMA cellular systems with multicell processing and Amplify-and-Forward cooperation between mobiles," IEEE Trans. Wireless Commun., vol. 6, no. 8, pp. 2942-2951, Aug. 2007.

[12] O. Somekh, O. Simeone, H. V. Poor and S. Shamai, "Cellular systems with full-duplex Amplify-and-Forward relaying and cooperative base stations," in Proc. IEEE International Symp. Inform. Theory (ISIT), Nice, France, June 24-29, 2007.

[13] O. Simeone, O. Somekh, Y. Bar-Ness and U. Spagnolini, "Throughput of low-power cellular systems with collaborative base stations and relaying," to appear in IEEE Trans. Inform. Theory.

[14] O. Simeone, O. Somekh, Y. Bar-Ness, H. V. Poor and S. Shamai (Shitz), "Capacity of linear two-hop mesh networks with rate splitting, decode-and-forward relaying and cooperation,” in Proc. Forty-Fifth Annual Allerton Conference on Communication, Control, and Computing, Monticello, Illinois, Sept. 26-28, 2007.

[15] G. Kramer, I. Maric and R. D. Yates, “Cooperative Communications,” Foundations and Trends in Networking (FnT), Now Publishers, Jun. 2007.
[16] A. D. Wyner, "Shannon-theoretic approach to a Gaussian cellular multiple-access channel," IEEE Trans. Inform. Theory, vol. 40, pp. 1713-1727, Nov. 1994.

[17] S. V. Hanly and P. A. Whiting, "Information-theoretic capacity of multireceiver networks,” Telecommun. Syst., vol. 1, pp. 1-42, 1993.

[18] Y. Lifang and A. Goldsmith, "Symmetric Rate Capacity of Cellular Systems with Cooperative Base Stations," in Proc. IEEE Globecom, Nov. 27- Dec. 1, San Francisco, California, 2006.

[19] Y. Lifang, T. Yoo and A. Goldsmith, "Coverage Spectral Efficiency of Cellular Systems with Cooperative Base Stations," in Proc. IEEE Globecom, Nov. 27- Dec. 1, San Francisco, California, 2006.

[20] O. Somekh, B. M. Zaidel, and S. Shamai (Shitz), "Sum rate characterization of joint multiple cell-site processing," IEEE Trans. Inform. Theory, vol. 53, pp. 4473-4497, Dec. 2007.

[21] S. Jing, D. N. C. Tse, J. Hou, J. Soriaga, J. E. Smee, and R. Padovani, "Multi-Cell Downlink Capacity with Coordinated Processing," in Proc. Information Theory and Application (ITA) Workshop, San Diego, California, Jan. 29 - Feb. 2, 2007.

[22] S. Jing, D. N. C. Tse, J. Hou, J. Soriaga, J. E. Smee, and R. Padovani, "Downlink macro-diversity in cellular networks," in Proc. IEEE International Symp. on Information Theory (ISIT'07), Nice, France, pp. 1-5, Jun. 24-29, 2007.

[23] A. Sanderovich, O. Somekh and S. Shamai (Shitz), "Uplink macro diversity with limited backhaul capacity,” in preparation.

[24] A. Sanderovich, S. Shamai, Y. Steinberg, and G. Kramer, "Communication via decentralized processing," to appear in IEEE Trans. Inform. Theory.

[25] S. Verdú, "Spectral efficiency in the wideband regime," vol. 48, no. 6, pp. 1329-1343, June 2002.

[26] S. Shamai and A. Wyner, "Information-theoretic considerations for symmetric cellular, multiple-access fading channels - part I,” IEEE Trans. Inform. Theory, vol. 43, no. 6, pp. 1877-1894, Nov 1997.

[27] O. Simeone, O. Somekh, S. Shamai (Shitz), and H. V. Poor, "Downlink macro-diversity with limited backhaul capacity," submitted.

[28] O. Simeone, O. Somekh, G. Kramer, H. V. Poor, and S. Shamai (Shitz), "Throughput of cellular systems with conferencing mobiles and cooperative base-stations," submitted to Eurasip Journal on Wireless Commun. and Networking, Aug. 2007.

[29] W. Yu, “Uplink-Downlink Duality via Minimax Duality,” IEEE Trans. Inform. Theory, vol. 52, no. 2, pp. 361-374, Feb. 2006.

[30] R. Dabora and S. Servetto, "Broadcast channels with cooperating decoders," IEEE Trans. Inform. Theory, vol. 52, no.12, pp. 5438-5454, Dec. 2006.

[31] S. C. Draper, B. J. Frey and F. R. Kschischang, "Interactive decoding of a broadcast message," Proc. Forty-First Annual Allerton Conference on Communication, Control, and Computing, Monticello, Illinois, 2003.

[32] Y. Liang and V. V. Veeravalli, "Cooperative relay broadcast channels," IEEE Trans. Inform. Theory, vol. 53, no. 3, pp. 900-928, March 2007.

[33] F. M. J. Willems, Informationtheoretical Results for the Discrete Memoryless Multiple Access Channel, Ph.D. thesis, Katholieke Universiteit Leuven, 1982

[34] I. Maric, R. D. Yates and G. Kramer, "The discrete memoryless compound multiple access channel with conferencing encoders,” in Proc. International Symposium on Inform. Theory (ISIT 2005), pp. 407-410, Adelaide, Australia, Sept. 4-9, 2005.

[35] C. T. K. Ng, I. Maric, A. J. Goldsmith, S. Shamai (Shitz), and R. D. Yates, "Iterative and one-shot conferencing in relay channels," in Proc. IEEE Information Theory Workshop (ITW 2006), Punta del Este, Uruguay, Mar. 13-17.

[36] A. Lapidoth, S. Shamai (Shitz) and M. Wigger, "A linear interference network with local side-information,” in Proc. International Symposium on Inform. Theory (ISIT 2007), Nice, France, June 24-29, 2007.

[37] D. Slepian and J. K. Wolf, "A coding theorem for multiple access channels with correlated sources," Bell Systems Tech. J., vol. 52, pp. 1037-1076, Sept. 1973. 